\title{
Preconditioning with PEP-1-SOD1 fusion protein attenuates ischemia/reperfusion-induced ventricular arrhythmia in isolated rat hearts
}

\author{
ZUNPING KE $^{1}$, AIMEI GAO ${ }^{2}$, PENG XU ${ }^{1}$, JIANING WANG $^{3}$, LIJUAN JI $^{4}$ and JIANYE YANG ${ }^{3}$ \\ Departments of ${ }^{1}$ Cardiology and ${ }^{2}$ Pharmacy, The Fifth People's Hospital of Shanghai, Fudan University, Shanghai 200040; \\ ${ }^{3}$ Institute of Clinical Medicine and Department of Cardiology, Hubei University of Medicine, Shiyan, Hubei 442000; \\ ${ }^{4}$ Department of Rehabilitation, The Second People's Hospital of Huai'an, Huai'an, Jiangsu 223001, P.R. China
}

Received August 4, 2014; Accepted April 13, 2015

DOI: $10.3892 / \mathrm{etm} .2015 .2440$

\begin{abstract}
PEP 1-Cu/Zn superoxide dismutase (PEP-1-SOD1) fusion protein preconditioning has been reported to protect the myocardium from ischemia/reperfusion (I/R)-induced injury by decreasing the infarct size, reducing levels of cardiomyocyte apoptosis and reducing the release of myocardial-specific biomarkers. The aim of the present study was to examine the effects of PEP-1-SOD1 pretreatment on I/R-induced ventricular arrhythmias in Langendorff-perfused rat hearts. The isolated rat hearts were pretreated with PEP-1-SOD1 prior to I/R, and the I/R-induced hemodynamic parameters, infarct size and ventricular arrhythmias were then assessed. Compared with the unprotected hearts, PEP-1-SOD1 preconditioning significantly improved the hemodynamic parameters, decreased the cardiac lactate dehydrogenase and creatine kinase-MB (CK-MB) levels, reduced the infarct size and attenuated the ventricular arrhythmia. Further investigation showed that PEP-1-SOD1 preconditioning reduced both the incidence and duration of ventricular tachycardia/ventricular fibrillation. In addition, the intracellular reactive oxygen species (ROS) levels were decreased. The results of the present study suggest that PEP-1-SOD1 preconditioning can protect the heart against I/R injury and attenuate I/R-induced arrhythmia by downregulating the generation of ROS.
\end{abstract}

Correspondence to: Professor Jianing Wang, Institute of Clinical Medicine and Department of Cardiology, Hubei University of Medicine, 39 Chaoyang Mid-Road, Shiyan, Hubei 442000, P.R. China

E-mail: rywjn@vip.163.com

Mrs. Lijuan Ji, Department of Rehabilitation, The Second People's Hospital of Huai'an, 62 South Huaihai Road, Huai'an, Jiangsu 223001, P.R China

E-mail: luzg1981@163.com

Key words: PEP-1-SOD1, ischemia/reperfusion, arrhythmia, reactive oxygen species

\section{Introduction}

Ischemic heart disease is one of the leading causes of morbidity and mortality worldwide (1). The survival of the ischemic myocardium is dependent upon early reperfusion; however, reperfusion is known as a 'double-edged sword' due to the spectrum of reperfusion-associated pathologies, which are collectively referred to as reperfusion injury (2). Ventricular arrhythmia is a potentially lethal consequence of myocardial ischemia/reperfusion (I/R) injury.

The administration of free radical scavengers and antioxidants has become an important approach in the treatment of myocardial ischemia or I/R injury and the suppression of ventricular arrhythmia. The key antioxidant enzyme $\mathrm{Cu} / \mathrm{Zn}$ superoxide dismutase (SOD1) acts to diminish the effects of oxidative stress by catalyzing the dismutation of the superoxide anion into hydrogen peroxide plus oxygen, thereby protecting the cells from oxidative damage; however, due to the considerable damage to the endogenous antioxidant activity induced by $\mathrm{I} / \mathrm{R}$, the vulnerability of the myocardium to oxygen free radicals is enhanced following I/R injury (3). Furthermore, the selectivity and limited permeability of the cell membrane prevents the delivery of exogenous SOD1 into living cells, thus limiting the ability of SOD1 to protect the cells/tissues from oxidative stress-induced damage (4).

PEP-1 is a peptide carrier that possesses the ability to deliver full-length, native peptides or proteins into cells without the requirement for conformational changes (5). Through protein transduction technology and the construction of full-length PEP-1 fusion proteins, numerous proteins, including enhanced green fluorescent protein, $\beta$-galactosidase, full-length specific antibodies, human copper chaperone for SOD1, catalase and SOD, have been successfully delivered into cultured cells and the nervous system (6-10). Our previous studies have indicated that PEP-1-SOD1 fusion proteins can be delivered into myocardial tissues to protect the myocardium from I/R-induced injury in rats (11-13); however, no studies, to the best of our knowledge, have considered the possible use of PEP-1-SOD1 as a therapeutic agent to treat the acute symptoms of myocardial ischemia or I/R injury-induced arrhythmia. The aim of the present 
study, therefore, was to evaluate the antiarrhythmic effects of PEP-1-SOD1 on myocardial I/R injury in rats subjected to transient coronary artery occlusion and reperfusion.

\section{Materials and methods}

Animals. Male Sprague Dawley rats (280-320 g) were obtained from Beijing HFK Bioscience Co. Ltd. (Beijing, China). The rats were kept in an animal house with a 12-h light/dark cycle. The study was approved by the Animal Ethics Committee of Hubei University of Medicine (Shiyan, China).

Generation of biologically active PEP-1-SOD1 fusion protein. PEP-1-SOD1 fusion protein was isolated and purified in accordance with the method described in one of our previous studies $(13,14)$. Briefly, two prokaryotic expression plasmids for SOD1 and PEP-1-SOD1 (Novogen, Billerica, MA, USA) were constructed using the TA-cloning method. Six histidine residues (His-tag; Promega, Madison, WI, USA) were used to tag the two recombinant at the amino terminus. The two proteins were expressed and purified separately, as previously described (14).

Induction of $I / R$. The rats were systemically heparinized [1,000 U/kg, intraperitoneal (i.p.)] and anesthetized with sodium pentobarbital (60 mg/kg, i.p.; Shanghai No.1 Biochemical and Pharmaceutical Co., Ltd., Shanghai, China). The heart was rapidly excised and a modified Langendorff apparatus was used to retrogradely perfuse the heart via the aorta under constant pressure $(80 \mathrm{mmHg})\left(95 \% \mathrm{O}_{2}\right.$ and $\left.5 \% \mathrm{CO}_{2}\right)$ with Krebs-Henseleit buffer $(118 \mathrm{mmol} / 1 \mathrm{NaCl}$, $24.5 \mathrm{mmol} / 1 \mathrm{NaHCO}_{3}, 4.7 \mathrm{mmol} / 1 \mathrm{KCl}, 1.2 \mathrm{mmol} / 1 \mathrm{KH}_{2} \mathrm{PO}_{4}$, $3.4 \mathrm{mmol} / 1 \mathrm{MgSO}_{4} \cdot 7 \mathrm{H}_{2} \mathrm{O}, 2.5 \mathrm{mmol} / 1 \mathrm{CaCl}_{2}$ and $5.5 \mathrm{mmol} / \mathrm{l}$ glucose; Shanhhai Zongze Biotechnology, Co., Ltd., Shanghai, China) at $37^{\circ} \mathrm{C}$, as previously described (15). A Kreb's solution-filled latex balloon was inserted into the left ventricle in order to monitor the left ventricular hemodynamic parameters using a digital acquisition and analysis system (Chengdu Taimeng Technology Co., Ltd., Chengdu, China). The hemodynamic parameters included heart rate (HR), left ventricular systolic pressure (LVSP) and the maximal rate of pressure rise (+dp/dtmax). Coronary flow (CF) was monitored by collecting pulmonary artery effluent. Following the recording of baseline data, each heart was subjected to $30 \mathrm{~min}$ of global ischemia, followed by $60 \mathrm{~min}$ of reperfusion. The elevation of the S-T segment on the electrocardiogram was indicative of the successful induction of ischemia.

Experimental groups. The rats were assigned to one of six groups (n=10/group), as follows: Sham (no global ischemia), control (subjected to global ischemia), PEP-1-SOD1-treated (25, 50 and $100 \mu \mathrm{mol} / \mathrm{l})$ and SOD1-treated $(100 \mu \mathrm{mol} / \mathrm{l})$. In the drug-treated groups, PEP-1-SOD1 and SOD1 were administered as components of the perfusion medium 15 min prior to ischemia. The hearts of the rats in the untreated group were perfused for an additional $15 \mathrm{~min}$ with perfusion medium only.

Measurement of cardiac marker enzymes. Coronary effluent was collected $60 \mathrm{~min}$ after reperfusion in order to measure the activity of lactate dehydrogenase (LDH) and serum creatine kinase-MB (CK-MB) using assay kits obtained from the
Nanjing Jiancheng Bioengineering Institute (Nanjing, China). Enzyme activity was determined spectrophotometrically.

Infarct size assessment. Myocardial infarct size was assessed with Evans Blue dye and 2,3,5-triphenyltetrazolium chloride (TTC) (Sigma-Aldrich, St. Louis, MO, USA). Following the excision of the heart, the left ventricle was cut into $2-\mathrm{mm}$ transverse slices, from apex to base. Following incubation in $1 \% \mathrm{TTC}(\mathrm{pH} 7.4)$ at $37^{\circ} \mathrm{C}$ for $15 \mathrm{~min}$, the slices were placed in $4 \%$ formaldehyde for 1 day. The infarcted myocardium was not stained by the TTC and thus appeared white, while the non-ischemic myocardium was stained brick-red by the TTC. Infarct area analyses were performed using ImageJ software (National Institutes of Health, Bethesda, MD, USA), and the results are expressed as the percentage of left ventricular volume for each heart.

Reperfusion-induced arrhythmia evaluation. Arrhythmias were evaluated based on the guidelines of the Lambeth Conventions (16). Ventricular tachycardia (VT) was defined as the occurrence of $\geq 4$ consecutive premature ventricular contractions, while ventricular fibrillation (VF) was defined as an inability to distinguish individual QRS deflections. The prevalence and total duration of VT and VF during the reperfusion period were measured.

Intracellular reactive oxygen species (ROS) measurement. ROS generation was measured with the sensitive fluorescent probe 2',7'-dichlorofluorescein diacetate (DCFH-DA; Nanjing Jiancheng Bioengineering Institute, Nanjing, China). Cardiomyocytes were dispersed from the hearts using digestion buffer, washed with KB solution and then incubated with $5 \mu \mathrm{mol}$ DCFH-DA for $20 \mathrm{~min}$, according to the instructions of commercial kits. The fluorescence intensity was measured using a fluorospectrophotometer with 488-nm excitation and 525-nm emission filters.

Statistical analysis. All experiments were performed in triplicate, and the data are presented as the mean \pm standard deviation. Statistical significance was determined using one-way analysis of variance. $\mathrm{P}<0.05$ was considered to indicate a statistically significant difference.

\section{Results}

Effects on hemodynamics. The LVSP, +dp/dtmax, CF and HR in the control group were observed to have decreased markedly after $60 \mathrm{~min}$ of reperfusion $(\mathrm{P}<0.01)$ (Table I). Compared with the control group, a significant recovery of LVSP, +dp/dtmax and CF was apparent in the PEP-1-SOD1-treated groups, and this effect was concentration-dependent $(\mathrm{P}<0.05)$. The rats in the SOD1 pretreated group had less obvious improvements in LVSP, +dp/dtmax and CF. No significant difference in HR was found between the control and pretreated groups.

Effects on $L D H$ and $C K-M B$ activity. The activity of the cardiac biomarkers in the coronary effluent was measured as an index of myocardial cellular injury following I/R and found to be elevated during reperfusion. As shown in Fig. 1, the LDH (Fig. 1A) and CK-MB (Fig. 1B) activities were significantly 


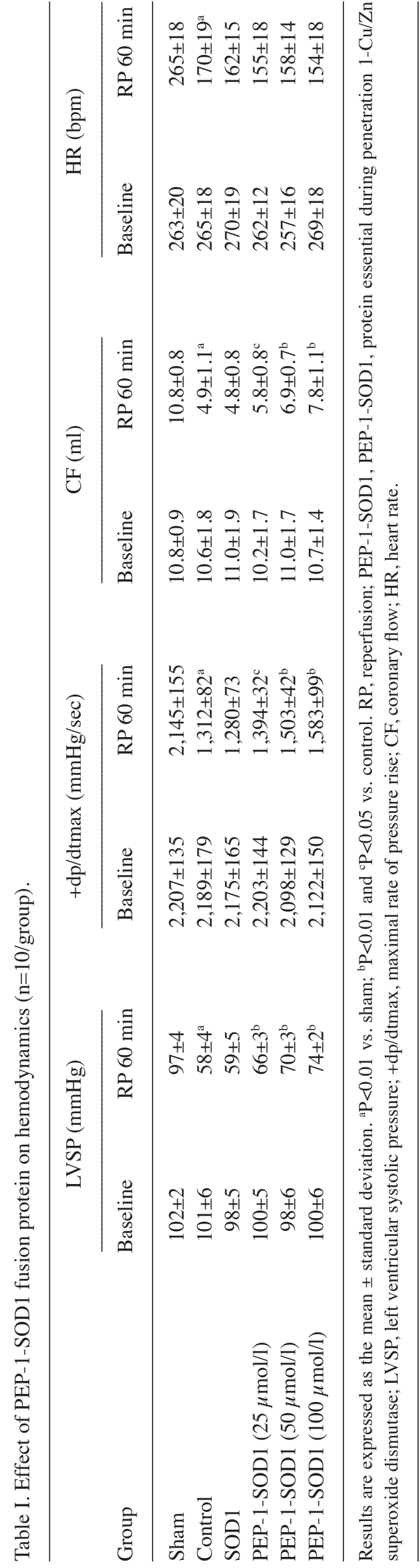

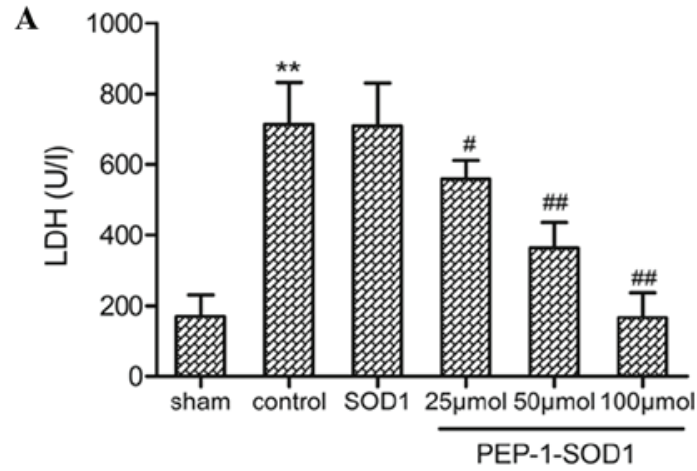

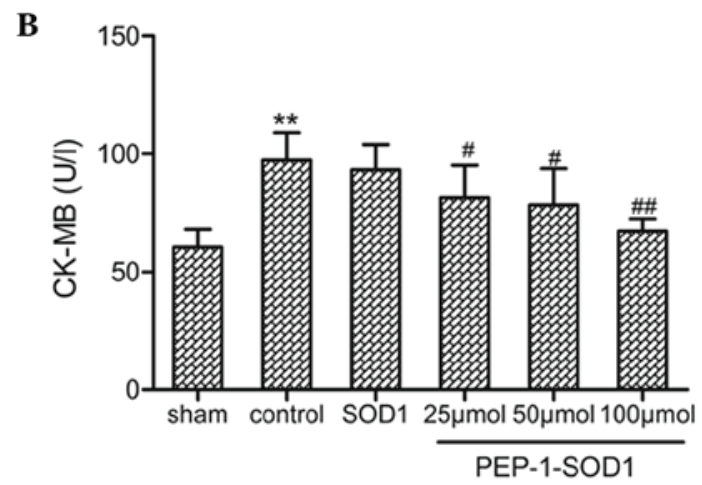

Figure 1. Effect of PEP-1-SOD1 fusion protein on (A) LDH and (B) CK-MB activity in coronary effluent after $30 \mathrm{~min}$ of ischemia followed by $60 \mathrm{~min}$ of reperfusion. Data are expressed as the mean \pm standard deviation $(n=10 /$ group). ${ }^{* *} \mathrm{P}<0.01$ vs. sham; ${ }^{\#} \mathrm{P}<0.05$ and ${ }^{\# \#} \mathrm{P}<0.01$ vs. control. PEP-1-SOD1, protein essential during penetration $1-\mathrm{Cu} / \mathrm{Zn}$ superoxide dismutase; $\mathrm{LDH}$, lactate dehydrogenase; $\mathrm{CK}-\mathrm{MB}$, serum creatine kinase-MB.
A

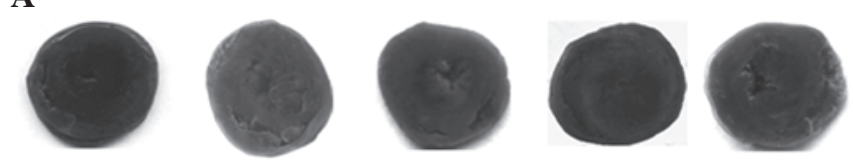

B

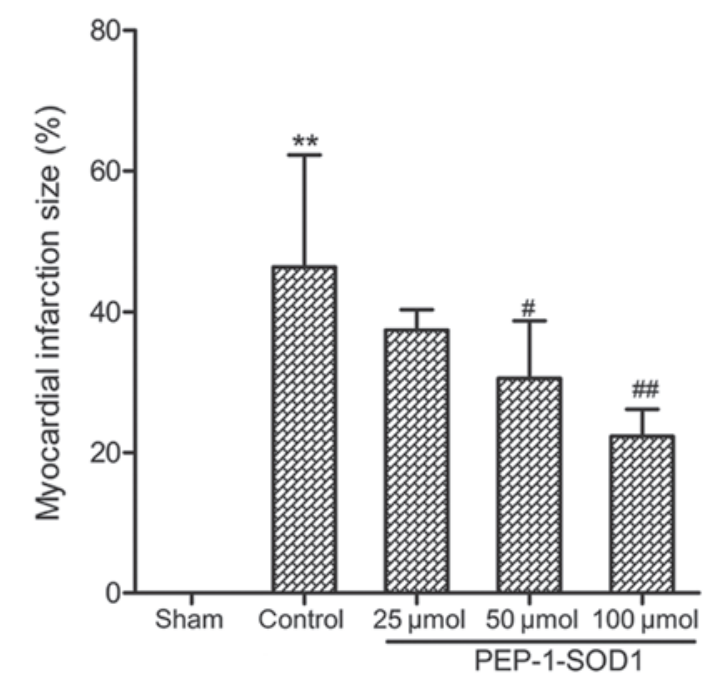

Figure 2. PEP-1-SOD1 fusion protein reduces myocardial infarction size. (A) 2,3,5-Triphenyltetrazolium chloride-stained myocardium $2 \mathrm{~h}$ after reperfusion. (B) Infarction size in each group. The infarct volume is expressed as a percentage of the left ventricular volume for each heart. Data are expressed as the mean \pm standard deviation ( $\mathrm{n}=10$ /group). ${ }^{* *} \mathrm{P}<0.01$ vs. sham; ${ }^{*} \mathrm{P}<0.05$ and ${ }^{\# \#} \mathrm{P}<0.01$ vs. control. PEP-1-SOD1, protein essential during penetration $1-\mathrm{Cu} / \mathrm{Zn}$ superoxide dismutase. 
A

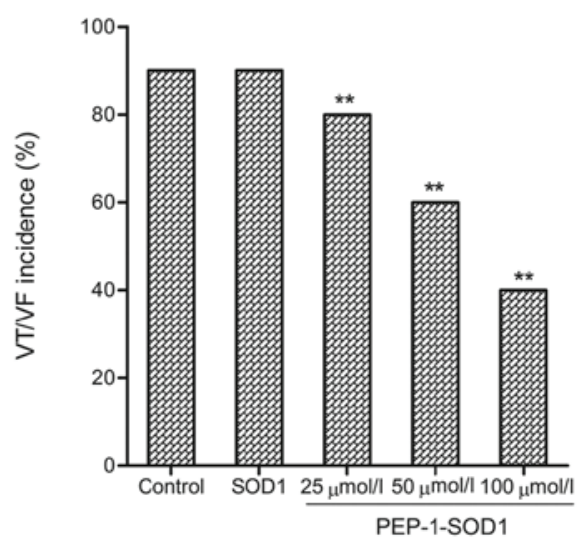

B

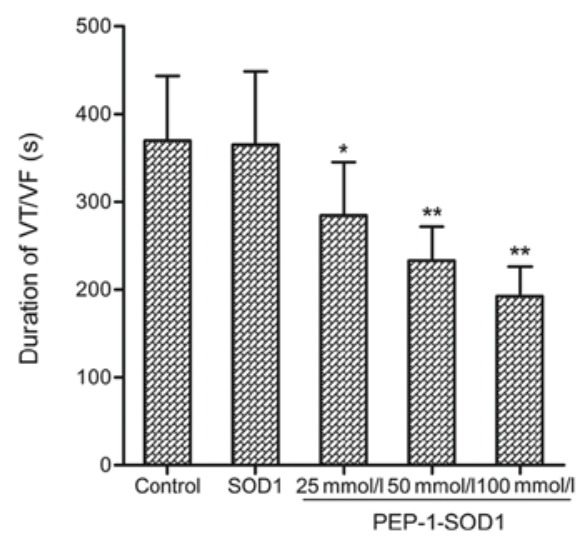

$\mathbf{C}$

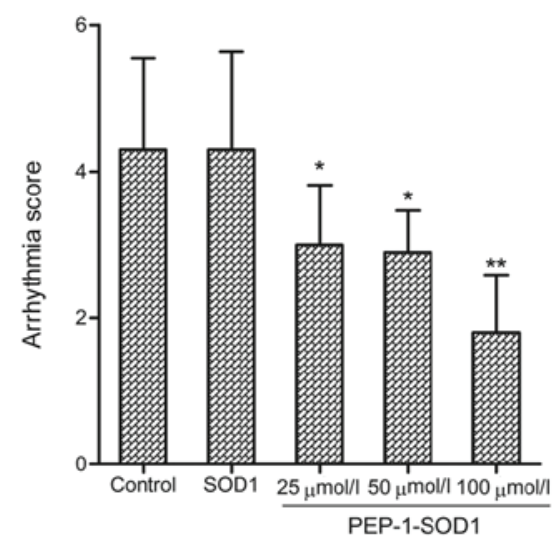

Figure 3. Effect of PEP-1-SOD1 on ischemia/reperfusion arrhythmias. The (A) incidence of VT/VF, (B) duration of VT/VF and (C) arrhythmia score in the reperfused hearts are shown separately. Data are expressed as the mean \pm standard deviation ( $\mathrm{n}=10$ /group). ${ }^{*} \mathrm{P}<0.05$ and ${ }^{* *} \mathrm{P}<0.01$ vs. control. PEP-1-SOD1, protein essential during penetration 1-Cu/Zn superoxide dismutase; VT, ventricular tachycardia; VF, ventricular fibrillation.

increased in the control group compared with those in the sham group. Pretreatment with PEP-1-SOD1 markedly decreased the LDH activity after $60 \mathrm{~min}$ of reperfusion compared with the control $(\mathrm{P}<0.05)$. Similar to the LDH activity, the CK-MB activity was also significantly reduced by PEP-1-SOD1 pretreatment $(\mathrm{P}<0.05)$.

Effect on infarct size. As shown in Fig. 2A and B, infarct size was significantly decreased in the PEP-1-SOD1-pretreated

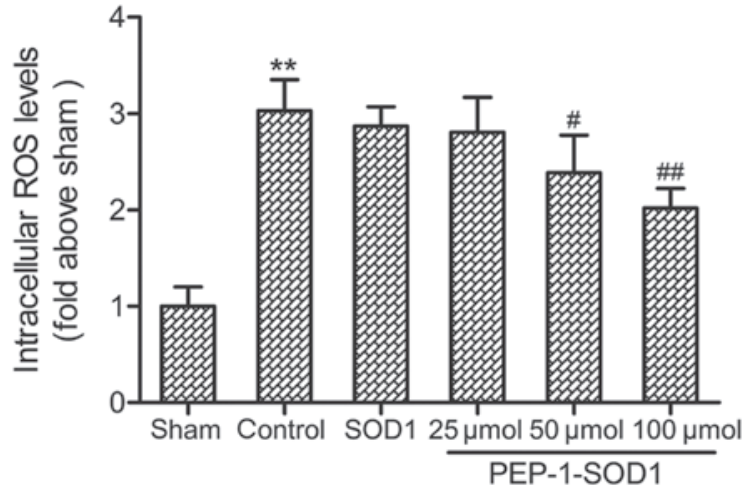

Figure 4. Effect of PEP-1-SOD1 on intracellular ROS levels. Data are expressed as the mean \pm standard deviation ( $n=10$ /group). ${ }^{* *} \mathrm{P}<0.01$ vs. sham; ${ }^{\#} \mathrm{P}<0.05$ and ${ }^{\# \#} \mathrm{P}<0.01$ vs. control. PEP-1-SOD1, protein essential during penetration $1-\mathrm{Cu} / \mathrm{Zn}$ superoxide dismutase; ROS, reactive oxygen species.

groups compared with that in the control group. The effect of PEP-1-SOD1 was concentration-dependent $(\mathrm{P}<0.05)$.

Effect on arrhythmias. To assess the I/R-induced arrhythmia, all ventricular arrhythmias that occurred during the 60-min reperfusion period were recorded. In the sham group, no obvious ventricular arrhythmias were noted. Compared with the control group, PEP-1-SOD1 significantly reduced both the duration and incidence of VT/VF (Fig. 3A and B). I/R-induced arrhythmias were additionally evaluated using a scoring system. The I/R-induced arrhythmia score was 4.3 in the control group; however, PEP-1-SOD1 administration was shown to significantly decrease this score (Fig. $3 \mathrm{C}, \mathrm{P}<0.05$ ).

Effect on intracellular ROS levels. It is generally accepted that ROS are one of the major contributors to I/R-induced myocardial damage; therefore, the effect of PEP-1-SOD1 on I/R-induced ROS generation was measured. As shown in Fig. 4, the intracellular ROS level in the control group increased to $303 \%$ of the level of the sham group. Significant reductions in ROS levels were observed in the PEP-1-SOD1-treated groups compared with the control group $(\mathrm{P}<0.05)$.

\section{Discussion}

Myocardial I/R injury can induce ventricular arrhythmia, leading to circulatory collapse and ultimately sudden mortality. The proposed underlying pathophysiological mechanisms include a burst generation of reactive oxygen intermediates, intracellular $\mathrm{Ca}^{2+}$ handling and ion channels. It is therefore believed that the effective inhibition of oxygen free radical production or the elimination of oxygen free radicals could reduce the ventricular arrhythmia caused by I/R injury (17). In the present study, the results showed that pretreatment with PEP-1-SOD1 led to a recovery in cardiac function (Table I), a reduction in myocardial-specific biomarker release (Fig. 1) and decreased infarct size (Fig. 2) in isolated rat hearts exposed to I/R injury. These findings were similar to those of previous studies $(11,13)$. In addition, it was found that PEP-1-SOD1 could attenuate reperfusion-induced arrhythmia, as shown by reductions in the VT/VF duration, VT/VF incidence and arrhythmia score (Fig. 3). 
At present, the precise mechanism underlying the antiarrhythmic effects elicited by PEP-1-SOD1 remains elusive. In this study, PEP-1-SOD1 was shown to reduce the myocardial I/R injury-induced ROS generation; the generation of ROS plays an important role in the regulation of reperfusion-induced arrhythmia. A recent study (18) reported that ion channels, such as the ATP-sensitive potassium channel, L-type calcium channel, $\mathrm{Na}^{+}-\mathrm{Ca}^{2+}$ exchanger and $\mathrm{Na}^{+}-\mathrm{H}^{+}$exchanger, also have critical functions in the pathophysiology and electrophysiology of I/R injury. It has yet to be determined whether these ion channels are involved in the modulation of reperfusion-induced arrhythmia by PEP-1-SOD1. These findings, in combination with those of our previous studies, indicate that the cardioprotective effects elicited by PEP-1-SOD1 are associated with the regulation of lipid peroxidation and ROS generation.

The present study was subject to several limitations. It should be noted that the study evaluated reperfusion-induced arrhythmia in the Langendorff model, in which the hearts were denervated and were not affected by extrinsic factors; however, reperfusion-induced arrhythmia is associated not only with the dysfunction of the intact hearts, but also with neurohumoral factors. The antiarrhythmic effects of PEP-1-SOD1 therefore still require verification in in vivo models.

In conclusion, this study has provided evidence that PEP-1-SOD1 can effectively improve the hemodynamic parameters, decrease the activity of myocardial-specific biomarkers, reduce the infarct size and downregulate ventricular arrhythmias in isolated rat hearts subjected to I/R insult. PEP-1-SOD1 reduces both the duration and incidence of $\mathrm{VT} / \mathrm{VF}$, and this effect may be associated with the regulation of lipid peroxidation and ROS generation.

\section{Acknowledgements}

This study was supported by the Hubei Province Outstanding Scientific Innovation Team Plans (grant no. T200811) and the Shiyan City Significant Technological Project (grant no. 2006030Z6).

\section{References}

1. Forini F, Nicolini G and Iervasi G: Mitochondria as key targets of cardioprotection in cardiac ischemic disease: Role of thyroid hormone triiodothyronine. Int J Mol Sci 16: 6312-6336, 2015.

2. Kalogeris T, Bao Y and Korthuis RJ: Mitochondrial reactive oxygen species: A double edged sword in ischemia/reperfusion vs. preconditioning. Redox Biol 2: 702-714, 2014.
3. Vijayasarathy K, Shanthi Naidu K and Sastry BK: Melatonin metabolite 6-Sulfatoxymelatonin, $\mathrm{Cu} / \mathrm{Zn}$ superoxide dismutase, oxidized LDL and malondialdehyde in unstable angina. Int J Cardiol 144: 315-317, 2010.

4. Zhang YE, Fu SZ, Li XQ, et al: PEP-1-SOD1 protects brain from ischemic insult following asphyxial cardiac arrest in rats. Resuscitation 82: 1081-1086, 2011.

5. Kim MJ, Jeong HJ, Kim DW, et al: PEP-1-PON1 protein regulates inflammatory response in raw 264.7 macrophages and ameliorates inflammation in a TPA-induced animal model. PLoS One 9: e86034, 2014.

6. He XH, Wang Y, Yan XT, et al: Transduction of PEP-1-heme oxygenase-1 fusion protein reduces myocardial ischemia/reperfusion injury in rats. J Cardiovasc Pharmacol 62: 436-442, 2013.

7. Dong X, Wang JN, Huang YZ, Guo LY and Kong X: Cell-penetrating peptide PEP-1-mediated transduction of enhanced green fluorescent protein into human colorectal cancer SW480 cells. Ai Zheng 26: 216-219, 2007.

8. Henriques ST, Costa J and Castanho MA: Translocation of betagalactosidase mediated by the cell-penetrating peptide pep-1 into lipid vesicles and human HeLa cells is driven by membrane electrostatic potential. Biochemistry 44: 10189-10198, 2005.

9. Kim W, Kim DW, Yoo DY, et al: Neuroprotective effects of PEP-1-Cu,Zn-SOD against ischemic neuronal damage in the rabbit spinal cord. Neurochem Res 37: 307-313, 2012.

10. Zhang L, Dong XW, Wang JN, et al: PEP-1-CAT-transduced mesenchymal stem cells acquire an enhanced viability and promote ischemia-induced angiogenesis. PLoS One 7: e52537, 2012.

11. Zhang YE, Wang JN, Tang JM, et al: In vivo protein transduction: Delivery of PEP-1-SOD1 fusion protein into myocardium efficiently protects against ischemic insult. Mol Cells 27: 159-166, 2009.

12. Ke ZP, Wang JN, Tang JM, et al: The role of PEP-1-SOD1 fusion protein on ischemia-reperfusion injury in isolated perfused rat hearts. Zhonghua Xin Xue Guan Bing Za Zhi 37: 268-274, 2009 (In Chinese).

13. Huang GQ, Wang JN, Tang JM, et al: The combined transduction of copper, zinc-superoxide dismutase and catalase mediated by cell-penetrating peptide, PEP-1, to protect myocardium from ischemia-reperfusion injury. J Transl Med 9: 73, 2011.

14. Wang JN, Ding P, Huang YZ, Luo LN, Guo LY, Kong X and Shao F: The protective effect of PEP-1-SOD1 preconditioning on hypoxia/reoxygenation injury in cultured human umbilical vein endothelial cells. Zhonghua Xin Xue Guan Bing Za Zhi 35: 750-756, 2007 (In Chinese).

15. Bhandary BI, Piao CS, Kim DS, Lee GH, Chae SW, Kim HR and Chae HJ: The protective effect of rutin against ischemia/reperfusion-associated hemodynamic alteration through antioxidant activity. Arch Pharm Res 35: 1091-1097, 2012.

16. Curtis MJ and Walker MJ: Quantification of arrhythmias using scoring systems: An examination of seven scores in an in vivo model of regional myocardial ischaemia. Cardiovasc Res 22: 656-665, 1988

17. Gong JS, Yao YT, Fang NX and Li LH: Sevoflurane postconditioning attenuates reperfusion-induced ventricular arrhythmias in isolated rat hearts exposed to ischemia/reperfusion injury. Mol Biol Rep 39: 6417-6425, 2012.

18. Lee JI, Wook Nha K, Suh JS, Choo SK, Park JH and Park JW: Remote postconditioning attenuates ischemia/reperfusion injury in rat skeletal muscle through mitochondrial ATP-sensitive $\mathrm{K}^{+}$channel-dependent mechanism. J Reconstr Microsurg 29: 571-578, 2013. 